\author{
Dagoberto Álvarez-Fernández • \\ Margarita Vela-Ramirez • Martha Rodríguez-Morán • \\ Fernando Guerrero-Romero
}

\title{
Clampless abdominal hysterectomy: a pilot study
}

Received: 22 June 2005 / Accepted: 23 December 2005 / Published online: 21 February 2006

(C) Springer-Verlag Berlin / Heidelberg 2006

\begin{abstract}
Our objective was to evaluate reduction in surgical bleeding by a modification in the customary simple abdominal hysterectomy (TAH) technique, the clampless abdominal hysterectomy (CAH). Eligible women were those with indications for abdominal hysterectomy. CAH is based on a simple distal tie-cut technique of the round ligaments, infundibulopelvic ligaments, and uterine vein and arteries, in comparison with the TAH technique of clamp-clamp-cut-tie-tie. Differences between the groups were tested by two-tailed unpaired Student $t$-tests (or the Mann Whitney $U$-test). Women in the CAH group $(n=19)$ showed significantly less surgical bleeding $(266.1 \pm 200.0$ vs. $502.5 \pm 441.4 \mathrm{ml}, p=0.04)$ and shorter surgical times ( $66.9 \pm 26.9$ vs. $145.0 \pm 50.9 \mathrm{~min}, p=0.000002)$ than women in the TAH $(n=21)$ group. We conclude that $\mathrm{CAH}$ could be a useful alternative for removing the uterus, significantly reducing both surgical bleeding and surgical time.
\end{abstract}

Keywords Abdominal hysterectomy · Technique · Surgical bleeding $\cdot$ Surgical time $\cdot$ Uterus $\cdot$ Clampless

D. Álvarez-Fernández

Surgical Oncology Department, General Hospital

of the Mexican Social Security Institute (MSSI),

Durango, Mexico

M. Vela-Ramirez

Gynecology Department, General Hospital of the Mexican

Social Security Institute (MSSI)

Durango, Mexico

M. Rodríguez-Morán · F. Guerrero-Romero $(\bowtie)$

Medical Research Unit in Clinical Epidemiology,

Mexican Social Security Institute (MSSI),

Durango, Mexico

e-mail: guerrero_romero@hotmail.com

Tel: : +52-618-8120997

Fax: +52-618-8132014

\section{Introduction}

Abdominal hysterectomy is undoubtedly the surgical intervention most frequently performed by gynecologists worldwide $[1,2]$. About $90 \%$ of hysterectomies are done to treat uterine leiomyomas, dysfunctional bleeding, uterine prolapse, and malignant diseases of the uterus [3]. Although hysterectomy can also be performed vaginally or laparoscopically or by laparotomy or mini-laparotomy, or by a combined approach of these techniques, total abdominal hysterectomy (TAH), which is relatively simple and safe, is still the most common surgical procedure [4] for removing the uterus in the presence of benign lesions and several malignant diseases $[3,5,6]$.

Because some people will not accept blood transfusions, which is a limitation for surgical procedures, the aim of this study was to evaluate the reduction in surgical bleeding by a modification in simple abdominal hysterectomy technique, the clampless abdominal hysterectomy (CAH).

\section{Materials and methods}

With approval of the protocol by the Institutional Research Committee and after obtaining the subjects' informed consent, a cross-sectional pilot study comparing the TAH and CAH groups was carried out from July 2004 to January 2005 in the General Hospital of the Mexican Social Security Institute in Durango, Mexico.

Eligible women (those with indications for abdominal hysterectomy) were randomly allocated to the TAH and CAH groups. Before randomization, women were clinically evaluated and laboratory tested to determine the presence of clinical contraindications for the surgical procedure, which were exclusion criteria. In addition, women receiving chemotherapy or radiotherapy were not included.

The primary trial end point of this pilot study was reduction in surgical bleeding. Sample size was estimated based on a statistical power of $80 \%$, alpha value of 0.05 , and an expected reduction in surgical bleeding by $50 \%$ for 
the women in the CAH group compared with those in the TAH group [8].

Thirty-nine eligible women were enrolled and randomly allocated to the TAH and $\mathrm{CAH}$ groups for surgical intervention. Computer-generated random numbers were used to assign participants to each intervention group. The final distribution was 21 women in the TAH group and 18 in the CAH group.

TAH was done according to the customary technique for abdominal hysterectomy [7]. CAH was done as follows:

Access to the uterus is through a vertical incision of the abdominal wall, similar to the TAH technique. Anatomic components of the uterus are identified, and two Kocher clamps are placed in the uterine cornus in a way that involves the uterus-ovarian ligament, round ligament, Fallopian tube, and the ascendant portion of the uterine artery and venous channels. By traction on the uterus, the pedicle is tensed, and with previous identification of the ureters, round ligaments on both sides are tied in a direct way (without clamping, using \#0 silk and squared knots) and cut. The infundibulopelvic ligaments on both sides also are distally sutured and cut in a direct way. The anterior leaf of the broad ligament is incised along the bladder reflection to the midline from both sides and the bladder dissected off the lower uterine segment and cervix. Finally, the uterine vein and arteries are identified, sutured, and cut in a direct way (Fig. 1). The second part of $\mathrm{CAH}$, for vaginal and abdominal wall closure, is similar to the customary technique used in TAH [8].

Bleeding was measured by pre- and postsurgical weighing of the gauzes and compresses used and quantifying the total blood aspirated.

Statistical analysis The preplanned intention-to-treat analysis of the primary study end point was done for all of the randomly allocated participants. Differences between the groups were assessed by two-tailed unpaired Student $t$-tests for comparison of normally distributed variables (or the Mann Whitney $U$-test for skewed data), or the chisquare test for categoric variables.

\section{Results}

Thirty-nine women who fulfilled the inclusion criteria were randomly allocated, 18 in the $\mathrm{CAH}$ and 21 in the TAH control group. None dropped out or had serious adverse events due to the surgical intervention. All the women in both groups satisfactorily completed the study and were included in the data analysis.

The overall average age was $44.0 \pm 12.1$ years. There was no significant age difference $(p=0.26)$ between the women in the CAH and TAH groups $(46.3 \pm 13.2$ vs. $41.9 \pm 10.6$ years, respectively).

Indications for hysterectomy in the group under study were uterine leiomyomas $(66.7 \%)$, cervical-uterine carcinoma in situ $(7.7 \%)$, dysfunctional uterine bleeding $(10.2 \%)$, endometrial carcinoma $(7.7 \%)$, pelvic organ prolapse $(2.6 \%)$, and ovarian carcinoma $(5.1 \%)$. There were no significant differences in uterine size $(p=0.34)$ between the CAH $\left(695.3 \pm 745 \mathrm{~cm}^{3}\right)$ and the TAH $(493.0 \pm$ $427.3 \mathrm{~cm}^{3}$ ) groups.

Women in the CAH group experienced significantly less operative blood loss $(266.1 \pm 200.0$ vs. $502.5 \pm 441.4 \mathrm{ml}$, $p=0.04)$ and lower operating times $(66.9 \pm 26.9$ vs. $145.0 \pm$ $50.9 \mathrm{~min}, p=0.000002)$ and anesthesia times ( $93.3 \pm 25.6$ vs. $172.8 \pm 52.4 \mathrm{~min}, p=0.000002)$ than women in the TAH group, whereas hospital stay was similar $(2.6 \pm 0.8$ vs. $2.7 \pm$ 0.7 days, $p=0.56$ ).

One patient in the TAH group and none in the CAH group had postsurgical bleeding that required reoperation.

\section{Discussion}

The proposed change in the surgical technique for removing a uterus simplifies the customary abdominal hysterectomy procedure. For cutting the round ligament, infundibulopelvic ligament, and vascular package, the usual technique of TAH involves the following steps: proximal clamp, distal clamp, cut, proximal suture, and distal suture. CAH simplifies this to distal suture and cut.

In 1950 Aldridge and Meredith [9] described a hysterectomy technique that eliminated the use of clamps, and Richardson and colleagues [10,11] later reported their experiences using a ligation technique and no crushing clamps. In addition to these previous experiences, our proposed changes to the customary technique are based on the anatomical distribution of the uterine circulation that makes possible the safe hemostasis of the uterine and ovarian arteries with clamping used for traction.
Fig. 1 Distal unique suture of round ligament (a), infundibulopelvic ligament (b), and vascular package of the uterus

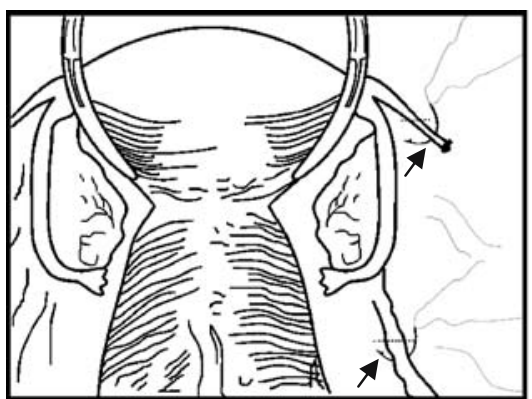

a

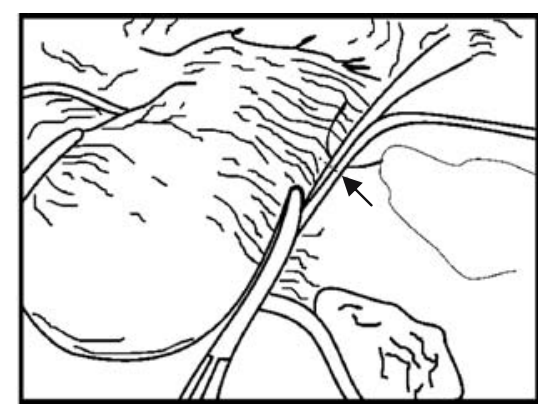

b 
We found that $\mathrm{CAH}$ reduced surgical time and, most importantly, reduced surgical bleeding $(266 \pm 200 \mathrm{ml}$, range 30-400 ml), which confers an opportunity for simple abdominal hysterectomy to those women who do not accept blood transfusions.

The claimed benefits of laparoscopic procedures for hysterectomy have the inconvenience of higher charges, longer operating times, and the use of expensive disposable instruments $[12,13]$. In this regard, $\mathrm{CAH}$ requires shorter surgical times and fewer surgical supplies, with the consequent reduction of facility costs and charges, facilitating access to this surgical procedure to women of lower socioeconomic groups.

In our sample, $66.7 \%$ of the surgical procedures were indicated by leiomyomas, and although the difference was not significant, uterine size was greater in the women in the $\mathrm{CAH}$ group, supporting the usefulness of the $\mathrm{CAH}$ technique for removing large uteri.

Finally, although the sample was sufficient to demonstrate significant differences in surgical bleeding and surgical time, it will be necessary to increase the sample size to convincingly demonstrate the safety of this modification to the Richardson technique.

In conclusion, the modified technique for abdominal hysterectomy that we propose significantly reduced surgical bleeding and could be a useful alternative for removing the uterus.

\section{References}

1. Farquhar CM (2002) Hysterectomy rates in the United States, 1990-1997. Obstet Gynecol 202:229-234
2. Materia E, Rossi L, Spadea T, Cacciani L, Baglio G, Cesaroni G, Arca M, Perucci CA (2002) Hysterectomy and socioeconomic position in Rome, Italy. J Epidemiol Community Health 56:461-465

3. Carlson KJ, Nicholas D, Schiff I (1982) Indications for hysterectomy. N Engl J Med 328:856-860

4. Weber AM, Lee J-Ch (1996) Use of alternative techniques of hysterectomy in Ohio, 1988-1994. New Engl J Med 335: 483-489

5. Livi L, Paiar F, Shah N, Blake P, Villanucci A, Amunni G, Barca R, Judson I, Lodge N, Meldolesi E, Simontacchi G, Piperono G, Galardi A, Scoccianti S, Biti GP, Harmer C (2003) Uterine sarcoma: twenty-seven years of experience. In J Radiation Oncol Biol 57:1366-1373

6. Schilling J, Wyss P, Faisst K, Gutzwiller F, Haller U (1999) For the Swiss Society of Gynecology and Obstetrics Switzerland. Gynecol Obstet 64:297-305

7. Richardson EH (1929) Simplified technique for abdominal panhysterectomy. Surg Gynecol Obstet 48:428

8. Mejía-Aranguré JM, Fajardo-Gutierrez A, Gómez-delgado A et al (1995) El tamaño de la muestra. Un enfoque práctico en la investigación clínica pediátrica. Bol Med Hosp Infant Mex 52:381-391

9. Aldridge AH, Meredith RS (1950) Complete abdominal hysterectomy, a simplified technique and end results in 500 cases. Am J Obstet Gynecol 59:748

10. Richardson AC, Lyon J, Graham EE (1973) Abdominal hysterectomy: Relationship between morbidity and surgical technique. Am J Obstet Gynecol 115:953-961

11. Richardson AC, Lyon JB (1981) The prevention of postoperative infection in abdominal hysterectomy. Clin Obstet Gynecol 24:1259-1266

12. Nezhat C, Bes O, Admón D, Nezhat CH, Nezhat F (1994) Hospital cost comparisons between abdominal, vaginal, and laparoscopic-assisted vaginal hysterectomies. Obstet Gynecol 83:713-716

13. Ellstrom M, Ferraz-Nunes J, Hahlin M, Olsson JH (1998) A randomized trial with a cost-consequence analysis after laparoscopic and abdominal hysterectomy. Obstet Gynecol 91:30-34 